\title{
Abdominal Ultrasonographic Findings in the Typhoid Fever in Pediatric Patients
}

\author{
M.M.El-Bakry ${ }^{1}$, A.M.Diab ${ }^{1}$, S.Z.Abdelaziz ${ }^{1}$ and B.M.El-Lobody ${ }^{2}$ \\ ${ }^{1}$ Pediatric,Pediatrics,Radiology Dept., Faculty of Medicine, Benha Univ., Benha, Egypt \\ ${ }^{2}$ (M.B.B.Ch), Faculty of Medicine, Benha Univ., Benha, Egypt \\ E-Mail:Basmaallobody@yahoo.com
}

\begin{abstract}
Typhoid fever is a fundamental irresistible illness of worldwide conveyance brought about by Salmonella Typhi. It is perhaps the most well-known bacterial reasons for intense febrile ailment in the creating scene. A complete analysis of typhoid fever is made by hemoculture just as the Widal test. USG is one of the demonstrative devices. The current examination was led to break down the handiness of USG in the determination of typhoid fever. This cross sectional examination was done on 50 pediatric patients matured 5-15 years who were clinically suspected to have typhoid fever. Blood tests of patients exposed to blood culture and Widal test and USG was acted in all patients. out of 50 patients Males were 27 cases and females were 23 cases. Out of 50 patients, 50 cases demonstrated splenomegaly on day 5 which diminished to 39 cases on day 10 and diminished to 29 cases on day 15 . Hepatomegaly was available in 20 cases on day 5 at that point diminished to 7 cases on day 10 at that point got negative on day 15 . Mesenteric lymphadenopathy was available in 21 cases on day 5 at that point diminished to 11 cases on day 10 at that point diminished to 6 cases on day 15 . Acalculus cholecystitis was available in 15 cases on day 5 at that point diminished to 9 cases on tenth day at that point perished to 5 cases on fifteenth day. Gut thickening was in 12 cases on day 5 at that point diminished to 7 cases on day 10 at that point got negative on day 15. USG is one of the solid indicative instruments in recognition of typhoid fever in youngsters. Highlights, for example, hepatomegaly, Splenomegaly, mesentric lymphadenopathy, acalculus cholecystitis and entrail thickening ought to be considered for the analysis of typhoid fever.
\end{abstract}

Keywords: Children, Typhoid fever, Ultrasonography.

\section{Introduction}

Typhoid fever is a fundamental irresistible sickness of worldwide conveyance brought about by salmonella enteric serotype typhi, stays a significant overall reason for grimness and mortality [1]. Typhoid fever is exceptionally infectious and spread through a human populace in territories with helpless water disinfection when polluted food or water is devoured [2]. The indications like fever, migraine, stoppage, disquietude, anorexia and loose bowels have been accounted for in typhoid fever however gut hole, encephalitis and demise have been accounted for just in extreme conditions [3]. For precise and dependable determination of typhoid fever is the utilization of blood culture technique ought to exceptionally be taken in thought [4]. Today enteric fever counteraction centers around improving sterilization, guaranteeing the security of food and water supplies, ID and the board of constant transporters of S.Typhi, and the utilization of typhoid antibodies to diminish the weakness of hosts to disease [5]. Ultrasound is speedy, efficient, non-obtrusive and powerful for diagnosing enteric fever when serological outcomes are tedious and in presence of negative societies [6]. In endemic zones ultrasound discoveries of splenomegaly, thickening of the ileum and cecum and numerous mesenteric hubs, with or without expanded thick walled nerve bladder is symptomatic of typhoid fever especially when serology is dubious and societies are negative or not accessible [7]. In endemic zones blend of sonographic discoveries are run of the mill enough to legitimize commencement of treatment and is genuinely protected to say that typical ultrasound assessment right off the bat over the span of febrile sickness precludes typhoid fever [8].

\section{Aim of the study}

For determining the abdominal Ultrasonographic findings in the course of the typhoid fever.

\section{Patients and methods}

This cross sectional study was conducted on 50 male and female children aged from 5-15 years from January 2020 to June 2020, where 50 children with fever who admitted to Benha Fever Hospital and pediatric department in Benha University Hospital., Qalyobia Governorate, Egypt.

\subsection{Ethical approval}

This research accepted by Research Ethics Committee (REC) of Faculty of Medicine, Benha University Ethics committee reference number MS 31.12.2019 All procedures performed in studies involving human participants were in accordance with the ethical standards of the institutional and / or national research committee A written informed consent was obtained from each patient after explaining all steps of this study.

\subsection{Inclusion criteria}

Age of children from 5 to 15 years. History of Fever $\geq 38$ and Fever lasting more than one week in suspected cases of typhoid fever. 


\subsection{Exclusion criteria}

Children below 5 years and above 15 years and Fever with known focus.

\subsection{Full history taking}

Each pediatric patient subjected to complete Personal history taking, the complaint, Present history and Past history.

\subsection{Full clinical examination including}

General examination of patients as general look examination of the patients, Vital signs as pulse, blood pressure, capillary filling time, respiratory rate and temperature.

Abdominal examination: Inspection, palpation, percussion and Auscultation.

Laboratory investigations: C.B.C, Serum CRP measurement, widal test and blood culture were done. Blood samples of patients were used for culture in xylose deoxycholate agar. All patients underwent routine widal test after 7 days of fever.
Abdomino pelvic Ultrasound: Appratus: GE logic - pro - p7 with Convex Probe with frequency of (3-5.5) MHZ.

Patient position: Mainly supine with oblique lateral position on demand.

All abdominal and pelvic organs will be examined in details. All data will be documented on real time examination with soft \pm hard copy photos. Time of Ultrasound examination: was done with selected patients on day 5, day 10 and day 15 of presentation of fever. Results were subjected to statistical analysis.

\section{Results}

50 pediatric patients with fever aged from 5-15 years were included in the study from Benha Fever Hospital and pediatric department in Benha University. Abdominal ultrasound was performed in all 50 patients on day 5, day 10 and day 15 of presentation of fever. Out of 50 pediatric patients males were 27 cases and females were 23 cases.

Table (1) Sex of the studied patients.

\begin{tabular}{llll}
\hline Variable & & No. $(\mathbf{N}=\mathbf{5 0})$ & $\mathbf{\%}(\mathbf{1 0 0 \%})$ \\
\hline Sex & Male & 27 & 54.0 \\
& Female & 23 & 46.0 \\
\hline
\end{tabular}

CBC findings among the studied patients. Anemia was present in $60 \%$ of cases, leucopenia in $66 \%$ of

Table (2) Findings of CBC among the studied patient. cases, neutropenia in $48 \%$ of cases, thrombocytopenia in $36 \%$ of cases, lymphocytosis in $38 \%$ of cases and eosinopenia in $12 \%$ of cases.

\begin{tabular}{lcc}
\hline Variable & No. $(\mathbf{N}=\mathbf{5 0})$ & \%( $\mathbf{1 0 0 \%})$ \\
\hline Anemia & 30 & 60.0 \\
Leucopenia & 33 & 66.0 \\
Neutropenia & 24 & 48.0 \\
Thrombocytopenia & 18 & 36.0 \\
Lymphocytosis & 19 & 38.0 \\
Eosinopenia & 6 & 12.0 \\
\hline
\end{tabular}

Symptoms among the studied patients.Fever was present in $100 \%$ of cases, abdominal pain in $66 \%$ of cases, vomiting in $38 \%$ of cases headache in $32 \%$ of

Table (3) Symptoms among the studied patients. cases, diarrhea in $32 \%$ of cases and constipation in $22 \%$ of cases. So most common symptoms were fever, abdominal pain and vomiting while less common symptoms were headache, diarrhea and constipation.

\begin{tabular}{|c|c|c|c|c|}
\hline Variable & & & No. $(\mathrm{N}=50)$ & $\%(100 \%)$ \\
\hline \multirow{12}{*}{ Symptoms } & \multirow[t]{2}{*}{ Fever } & Negative & 0 & 00.0 \\
\hline & & Positive & 50 & 100.0 \\
\hline & \multirow[t]{2}{*}{ Abdominal pain } & Negative & 17 & 34.0 \\
\hline & & Positive & 33 & 66.0 \\
\hline & \multirow[t]{2}{*}{ Headache } & Negative & 34 & 68.0 \\
\hline & & Positive & 16 & 32.0 \\
\hline & \multirow[t]{2}{*}{ Vomiting } & Negative & 31 & 62.0 \\
\hline & & Positive & 19 & 38.0 \\
\hline & \multirow[t]{2}{*}{ Diarrhea } & Negative & 32 & 64.0 \\
\hline & & Positive & 18 & 36.0 \\
\hline & \multirow{2}{*}{ Constipation } & Negative & 39 & 78.0 \\
\hline & & Positive & 11 & 22.0 \\
\hline
\end{tabular}


Out of 50 patients, 50 cases showed splenomegaly on day 5 which decreased to 39 cases on day 10 and decreased to 29 cases on day 15 . Hepatomegaly was present in 20 cases on day 5 then decreased to 7 cases on day 10 then became negative on day 15 . Mesenteric lymphadenopathy was present in 21 cases on day 5 then decreased to 11 cases on day 10 then decreased to 6 cases on day 15. Acalculus cholecystitis was present in 15 cases on day 5 then decreased to 9 cases on day 10 then deceased to 5 cases on day 15 . Bowel thickening was in 12 cases on day 5 then decreased to 7 cases on day 10 then became negative on day 15 .

Table (4) Ultrasound findings among the studied patients over the period of follow up.

\begin{tabular}{lccccc}
\hline Follow up & Hepatomegaly & Splenomegaly & $\begin{array}{c}\text { Mesentric } \\
\text { Lymphadenopathy }\end{array}$ & $\begin{array}{c}\text { Acalculus } \\
\text { Cholecystitis }\end{array}$ & $\begin{array}{c}\text { Bowel } \\
\text { thickening }\end{array}$ \\
\hline Day 5 & 20 & 50 & 21 & 15 & 12 \\
Day 10 & 7 & 39 & 11 & 9 & 7 \\
Day 15 & 0 & 29 & 6 & 5 & 0 \\
\hline
\end{tabular}
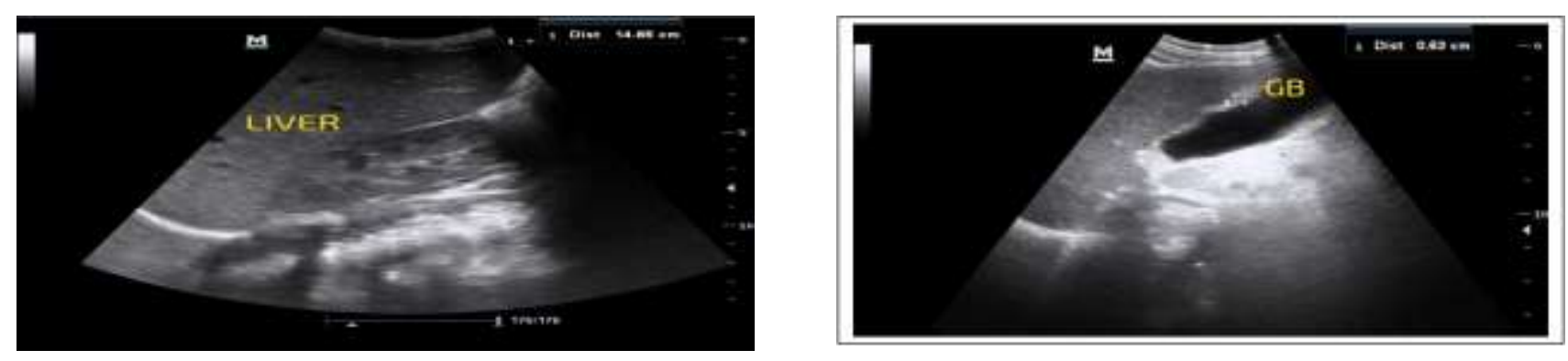

Fig (1) Abdominal ultrasonography (USG) findings for typhoid fever in children. (A) Hepatomegaly. (B) Thick gall bladder.

\section{Discussion}

Typhoid fever brought about by salmonella typhi, it is endemic in low-and center pay nations, Africa and Asia were recognized as high-endemic nations for typhoid fever [9].

In the current investigation, out of 50 patients guys were 27 cases and females were 23 cases, these outcomes come in concurrence with [10] who found that guys are more influenced in typhoid fever than females, additionally detailed this outcomes. However, these outcomes come interestingly with [11] who found that females were more influenced than guys.

We found that 50 cases $(100 \%)$ of patients had fever this is in concurrence with [6] who announced fever in $(100 \%)$ of his cases. These outcomes came conversely with [12] who discovered fever in $(75.8 \%)$ of his cases.

We found that 16 cases (32\%) of patient gathering had migraine. This outcome came in concurrence with [13] who revealed cerebral pain in $(32 \%)$ of cases. these outcomes came interestingly with [14] who discovered cerebral pain in $(58 \%)$ of their cases.

We discovered 33 cases $(66 \%)$ of the patient gathering had stomach torment this outcome come in concurrence with [15] who discovered stomach torment in $(68 \%)$ of patients. Then again, these outcomes came conversely with [16] who discovered stomach torment in $(19 \%)$ of their cases.

We discovered 18 cases (36\%) of patients had looseness of the bowels, this outcome came in concurrence with [17] who discovered the runs in (37\%) of his cases, the outcome came interestingly with (16) who discovered the runs in (14\%) of their cases and conversely with [12] who discovered the runs in (74.2\%) of his cases

We found that 19 cases $(38 \%)$ of patients had regurgitating. The heaving rate was more than that announced by [6] who found that spewing in $(6.7 \%)$ of cases and not exactly that detailed by [13] who revealed retching in $(52 \%)$ of his cases.

We discovered sickliness in 30 cases $(60 \%)$ of patients, this examination was in concurrence with (18) who discovered pallor in $(63.7 \%)$ of his cases On the other hand [19] demonstrated no critical contrast with respect to hemoglobin level in his investigation as his cases change with age, were gentle and he done his examinations in the first seven day stretch of sickness.

We found that out of 50 patients, 50 cases indicated splenomegaly on day5 which diminished to 39 cases on day10 then got negative on day 15 . Hepatomegaly was in 20 cases on day 5 then diminished to 7 cases on day 10 at that point got negative on day 15 this outcomes came in concurrence with [20] who discovered 38 of 38 patients had splenomegaly on day 5 then diminished to 30 cases on day 10 then became 22 cases on day 15 , he additionally found that 15 of 38 patients had hepatomegaly on day 5 at that point diminished to 5 cases on day10 then got negative on day 15 . This outcome likewise came conversely with [21] who found that 40 of 60 cases had splenomegaly on day 5 at that point diminished to 32 cases on day10 then to 20 cases on day 15 , he additionally discovered hepatomegaly in 18 of 60 cases on day 5 then diminished to 9 cases on day 10then got 
negative on day15. Acalculus cholecystitis was available in 15 cases on day 5 then diminished to 9 cases on day 10 at that point expired to 5 cases on day 15 , this outcome came in concurrence with [6] who discovered 9 of 30 patients had acalculus cholecystitis. This outcome came interestingly with [20] who found that 20 of 38cases had acalculus cholecystitis day 5 at that point diminished to 14 cases on day10 then got negative on day 15 .

\section{Conclusion}

Ultrasonography is one of the solid analytic instruments in the location of the typhoid fever in kids. Splenomegaly, hepatomegaly, developed mesenteric lymph hubs (MLNs), gut divider thickening and acalculus cholecystitis, are the stomach ultrasonographic discoveries in typhoid fever in pediatric patients.

\section{Recommendations}

The clinical and the ultrasonographic discoveries of amplified mesenteric lymph hubs, inside divider thickening, enlarged thick divider nerve bladder and biliary slime are helpful for determination of typhoid fever in any event, when widal test is uncertain and blood societies are sterile or not accessible.

\section{References}

[1] N.Crum. Estimating the incidence of typhoid fever and other febrile illnesses in developing countries. Emerg. Infect. Diseases, Vol.9(5), PP.539$544,2013$.

[2] E.J.Klemm, S.Shakoor, A.J.Page, F.N.Qamar. Emergence of an Extensively Drug- Resistant Salmonella enterica serovar Typhi clone Harboring a promiscuous plasmid Encoding Resistance to fluoroquinolones and third-generation cephalosporins. MBio, Vol.9(1), PP.105,2018.

[3] U.Ayub, A.A.Khattak, A.Saleem, F.Javid . Incidence of typhoid fever in Islamabad, Pakistan. Am-Eurassian Journal Toxical Sci, Vol.7(4), PP.220-223. ,2015.

[4] V.Mogasale, E.Ramani, V.V.Mogasale, J.Park . What proportion of Salmonella typhi cases are detected by blood culture?A systemic literature review. Annals of Clinical Microbiology Antimicrobials, Vol.15(1), PP.32, 2016.

[5] M.E.Carey, Z.I.Diaz, A.K.Zaidi, A.D.Steele A Global Agenda for Typhoid Control-A Perspective from the Bill \& Melinda Gates Foundation. Clin Infect Dis, Vol. 68(1), PP.42-45,2019.

[6] K.Ahsan, H.Begum, M.Ahsan, S.Momtaj. Abdominal ultrasonogram I Typhoid Fever: A Useful Diagnostic Tool. J.,pediatric Surgeon of Bangladesh, Vol.5(2), PP.58-63,2016.

[7] M.A.Mateen, S.Saleem , P.C.Rao. Ultrasound in the diagnosis of typhoid fever.Indian .J. Pediatr, Vol. 73(8), PP. 681-685,2006.

[8] A.Kobayashi, Y.Adachi, Y.Iwata, Y.Sakai. Abdominal Ultrasonographic Findings In Typhoid Fever: A Comparison Between Typhoid Patients
And Those With Non-Typhoidal Salmonella And Campylobacter Jejuni Enterocolitis Southeast Asian J Trop Med Public Health, Vol.43 (2), PP. 423-430,2012.

[9] N.Muresu, G.Sotgiu, A.Cossu, C.Cocuzza. TravelRelated Typhoid Fever: Narrative Review of the Scientific Literature Int. J. Environ. Res. Public Health, Vol.17(2), PP.615,2020.

[10]F.Essa, S.Hussain, D.Batool, A.Usman. Study of Socio-Demographic Factors affecting the prevalence of Typhoid Ann Med Health Sci Res, Vol. 9, PP.469-471,2019.

[11] A.Fusheini , S.K.Gyawu. Prevalence of typhoid and paratyphoid fever in the Hohoe Municipality of the Volta Region, Gana: A Five-Year Retrospective Trend Analysis.Annals of Global Health, Vol. 86(1), PP.111,2020.

[12] R.Kumar , N.Gupta , Multidrug-resistant typhoid fever. The Indian J.,Pediatrics, Vol.74(1), PP.3942,2007.

[13] K.G.Singh , S.S.Tunapudi. A Study on clinical profile of typhoid fever at Government General Hospital, Nizamabad, Telanagana, India. International Journal Contemp Pediatr, Vol.6, PP.2642-2645,2019.

[14]F.C.Barret, J.D.Knudsen, I.S.Johansen. Cases of typhoid fever in Copenhagen region: a retrospective study of presentation and relapse BMC research Notes, Vol. 6(1), PP.315,2013.

[15]M.Sohail, J.Masood, J.Khalid, Z.Anjum . XDR(Drug Resistant) typhoid fever in children. The Professional Medical Journal, Vol. 27(9), PP.19471951. 2020.

[16]B.Sahay ,A.Irfan. Evaluation of clinicodemographic and biochemical profile of pediatric patients diagnosed with typhoid fever. International J.,Health and Clinical Research, Vol.3(9), PP.227232,2020 .

[17]Z.Memon, P.Alfred, M.Rifkin. Health care preferences for children with typhoid fever in two slum communities in Karachi, Pakistan. Infect. Dis.J, Vol.39(6), PP.204-208,2008.

[18] A.Dangane, J.Ajobiewe , A.Nuhu. Haematological changes associated with Salmonella typhi and Salmonella paratyphi in humans Biomed Health. Sci, Vol.6(4), PP.243-47,2010.

[19] M.Parry, W.Lalith , B.Stephen. The utility of diagnostic tests for enteric fever in endemic locations. Expert Rev. Anti- Infect, Vol. 9(6), PP.711-725. ,2005.

[20]P.S.Patil ， P.L. Jadhav. Usefulness of Ultrasonography in detection of cases of typhoid fever in children- A clinical study. J.,Advanced Medical and Dental Sciences Research, Vol.5(11), PP. 96-98,2017.

[21] J.Anthony. Ultrasonography in diagnosis of typhoid fever in children: A radiological study. International J.,Radiology and Diagnostic Imaging, Vol.1(1), PP.4-6,2018. 